\title{
An early diagnosis of Mondini-like dysplasia in the Emergency Department at the first episode of bacterial meningitis
}

\author{
Jeong Ho Kang, ${ }^{1,2}$ Sung Wook Song, ${ }^{1,2}$ Sung Kgun Lee, ${ }^{1,2}$ Seo Young Ko, ${ }^{3}$ Ji Hwan Bu ${ }^{3}$ \\ ${ }^{1}$ Department of Emergency Medicine, School of Medicine, Jeju National University, Jeju; ${ }^{2}$ Institute for \\ Medical Science, Jeju National University, Jeju; ${ }^{3}$ Department of Emergency Medicine, Jeju National \\ University Hospital, Jeju, Republic of Korea
}

\begin{abstract}
Mondini-like dysplasia is a rare congenital inner ear malformation (IEM) and the most common anomaly associated with recurrent bacterial meningitis in children. Early diagnosis of Mondinilike dysplasia is crucial for preventing recurrent bacterial meningitis. We report a case of a 15-year-old girl with unrecognized Mondini-like dysplasia that was early diagnosed in the Emergency Department at the first episode of bacterial meningitis. Assessing hearing function in children who have their first episode of meningitis is crucial. Abnormal hearing test results are critical diagnostic findings that help to suspect an IEM. In addition, high-resolution computed tomography of the temporal bones is an excellent diagnostic tool for IEM.
\end{abstract}

\section{Introduction}

Bacterial meningitis is a life-threatening infectious disease, even though vaccination programs against Haemophilus influenzae type b and Pneumococcus have been introduced. Despite appropriate treatment, high mortality rate and long-term neurological

Correspondence: Sung Wook Song, Department of Emergency Medicine, School of Medicine, Jeju National University, 15, Aran 13Gil, Jeju-si, Jeju-do, 63241, Republic of Korea.

Tel. +82.64.717.1924 - Fax: +82.64.717.1168.

E-mail: sungwook78@gmail.com

Key words: Mondini dysplasia; Inner ear malformation; Meningitis; Incomplete partition.

Contributions: JHK, SWS, manuscript writing and references search; SKL, SYK, JHB, manuscript reviewing.

Conflict of interest: the authors declare no potential conflict of interest.

Funding: none.

Received for publication: 25 May 2019.

Revision received: 21 August 2019.

Accepted for publication: 17 September 2019.

This work is licensed under a Creative Commons Attribution 4.0 License (by-nc 4.0).

OCopyright: the Author(s), 2019

Licensee PAGEPress, Italy

Emergency Care Journal 2019; 15:8306

doi:10.4081/ecj.2019.8306 sequelae remain a significant concern. ${ }^{1}$ Recurrent bacterial meningitis is defined as the occurrence of two or more distinct episodes of meningitis, with the subsequent episode occurring after full recovery from the previous episode. ${ }^{2}$ Recurrence of bacterial meningitis is rare, occurring in only $1.3 \%$ of children and $9 \%$ of adults who have had a prior episode of this disease. This recurrence phenomenon is rare, occurring in $1.3 \%$ of affected children and $9 \%$ of affected adults, but raises a diagnostic challenge for clinicians to ascertain the pathology linked to recurrent bacterial meningitis because the early detection of any pathology can prevent further occurrences of meningitis. ${ }^{1}$ Mondini-like dysplasia is a uncommon congenital inner ear malformation (IEM) and this anomaly is most commonly associated with cerebrospinal fluid (CSF) leakage resulting from an anatomical defect of the temporal bone in children. Thus, it is a critical predisposing factor of recurrent bacterial meningitis. ${ }^{2-7}$ However, establishing a diagnosis of Mondini-like dysplasia is difficult, and a diagnosis is usually made after the occurrence of recurrent bacterial meningitis. We report a case of a young girl with unrecognized Mondini-like dysplasia, diagnosed very early in the Emergency Department (ED) at the first episode of bacterial meningitis.

\section{Case Report}

A 15-year-old girl visited our ED in January 2016 complaining of febrile sensation, headache, and neck pain, as well as right ear pain on the day before the presentation. Her past medical records revealed that she was born at the full term of pregnancy without perinatal problems. Her development and growth were unremarkable, except she was hard of hearing in the right ear. She had no admission history of meningitis or similar episodes.

At the time of arrival to the ED, her body temperature was 38.9 ${ }^{\circ} \mathrm{C}$, blood pressure was $138 / 77 \mathrm{mmHg}$, pulse rate was 105 beats per minute, and respiratory rate was 20 breaths per minute. Physical examination revealed an injected bulging right tympanic membrane with effusion and positive meningeal irritation signs. In initial laboratory tests, white blood cell count (WBC) was elevated at $20,900 / \mu \mathrm{L}$ with a predominance of neutrophils $(87.1 \%$ neutrophils, $8.4 \%$ lymphocytes, and $4.5 \%$ monocytes) in the differential count. The high-sensitive C-reactive protein level was slightly elevated at $0.42 \mathrm{mg} / \mathrm{dL}$. The results of other laboratory tests were within normal limits. Her consciousness level was alert at first, but it deteriorated to drowsy over time. We have taken brain computed tomography (CT) to distinguish the cause of sudden change in the consciousness of the patient. Brain CT findings showed no specific structural lesions in the brain parenchyma, but there was fluids collection in the right tympanic cavity, mastoid air cells. These findings suggested right-sided otomastoiditis and questionable inner ear pathologies. After confirming the result of brain CT, the lumbar 
puncture was performed immediately. Lumbar puncture yielded turbid, colorless fluid with an opening pressure of $320 \mathrm{~mm}$ of water. CSF analysis revealed a WBC count of $1440 / \mu \mathrm{L}$ ( $94 \%$ polymorphonuclear leukocytes and 6\% mononuclear cells), an elevated protein level of $164 \mathrm{mg} / \mathrm{dL}$, and a low glucose level of $35 \mathrm{mg} / \mathrm{dL}$ compared to a serum glucose level of $131 \mathrm{mg} / \mathrm{dL}$. We diagnosed bacterial meningitis with CSF analysis and administered empirical antibiotics to the patient.

Through the patient's hearing impairment history and brain CT results, we strongly suspected the inner ear pathologies associated with bacterial meningitis and conducted high-resolution computed tomography (HRCT) of the temporal bone. HRCT of the temporal bones revealed several abnormal findings in the right inner ear whereas no remarkable findings were noted in the left ear: incomplete partition of cochlea (absence of the modiolus and interscalar septum), hypoplasia of vestibular aqueduct, enlarged vestibule, semicircular canal dysplasia, and accumulation of fluids in the tympanic cavity and aircells. These abnormalities involving the right inner ear structure were consistent with Mondini-like dysplasia. (Figure 1) She was finally diagnosed with bacterial meningitis accompanied by unrecognized Mondini-like dysplasia at the ED. We consulted to the pediatrician, and the patient was admitted to the pediatric intensive care unit.

During her hospitalization, her condition improved remarkably, and she was transferred to the pediatric general ward five days after empirical antibiotics with cefotaxime and vancomycin. Blood cultures were negative, whereas CSF cultures eventually grew abundant penicillin-resistant Streptococcus pneumoniae. Besides, pure tone audiometry (PTA) and auditory brainstem response (ABR) evaluation were performed to evaluate the hearing function of the right ear during the hospitalization. PTA revealed a severe sensorineural hearing loss (SNHL) in the right ear, whereas the left ear showed normal threshold ranges (Figure 2). ABR analysis showed normal brainstem responses in the left ear with an increase in the click stimulus rate and no responses in the right ear to $90-\mathrm{dB}$ normal hearing level (nHL) click stimuli, suggestive of right retrocochlear pathology (Figure 3).

She had a two-week course of parenteral antibiotics, and no microorganism growth was noted on follow-up CSF cultures. Based on the results of previous ear tests, a pediatrician consulted an otolaryngologist and planned to undergo surgical treatment for Mondini-like dysplasia after meningitis was cured. The patient showed complete recovery; thus, she was discharged on the 20th day of hospitalization with prescribed oral antibiotics. An antipneumococcal vaccine was administered at the pediatric outpatient department 5 days after being discharged, and she underwent surgical treatment for Mondini-like dysplasia three months after meningitis was cured.

\section{Discussion}

Mondini dysplasia was first reported in 1791 by Carlo Mondini, described initially as triad of inner ear malformations; 1.5 turns of the cochlea (compared with the normal 2.5 turns) comprising a normal basal turn and a cystic apex, an enlarged vestibule with normal semicircular canals, and an enlarged vestibular aqueduct containing a dilated endolymphatic sac. ${ }^{8}$ Nowadays, various malformations involving the inner ear have been reported and the original Mondini dysplasia was regarded one of several variants of IEMs. However, until recent years the term of Mondini-like dysplasia has been usually used to refer to these IEMs. ${ }^{9}$
In terms of surgical approach, it is crucial to classify IEMs more accurately, so a radiologic classification of IEMs is recently presented based on differences in cochlear anatomy: 1, complete labyrinthine aplasia; 2 , rudimentary otocyst; 3 , cochlear aplasia; 4, common cavity; 5 , cochlear hypoplasia; 6 , incomplete partition of the cochlear; 7, enlarged vestibular aqueduct; 8 , cochlear aperture abnormalities. ${ }^{9}$ Based on the HRCT findings in our patient, we suggest that our patient had an incomplete partition type I (cystic cochleovestibular malformation).

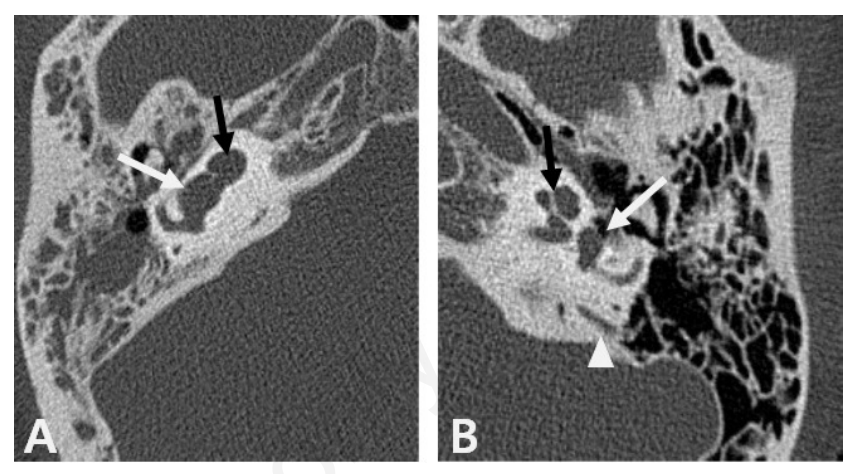

Figure 1. High-resolution computed tomography of the temporal bones of the patient. A) Right ear - Cochlea (black arrow) without modiolus and interscalar septum appears as an empty cystic structure accompanied by an enlarged vestibule (white arrow). In addition, fluid collection in the tympanic cavity and air cells suggests accompanying inner ear infection. B) Left ear - Cochlea (black arrow) shows a normal structure with modiolus and interscalar septum, and vestibule (white arrow) show a normal shape. Vestibular aqueduct (arrow head) shows normal in the left ear, but it is not clearly observed due to hypoplasia in the right ear.

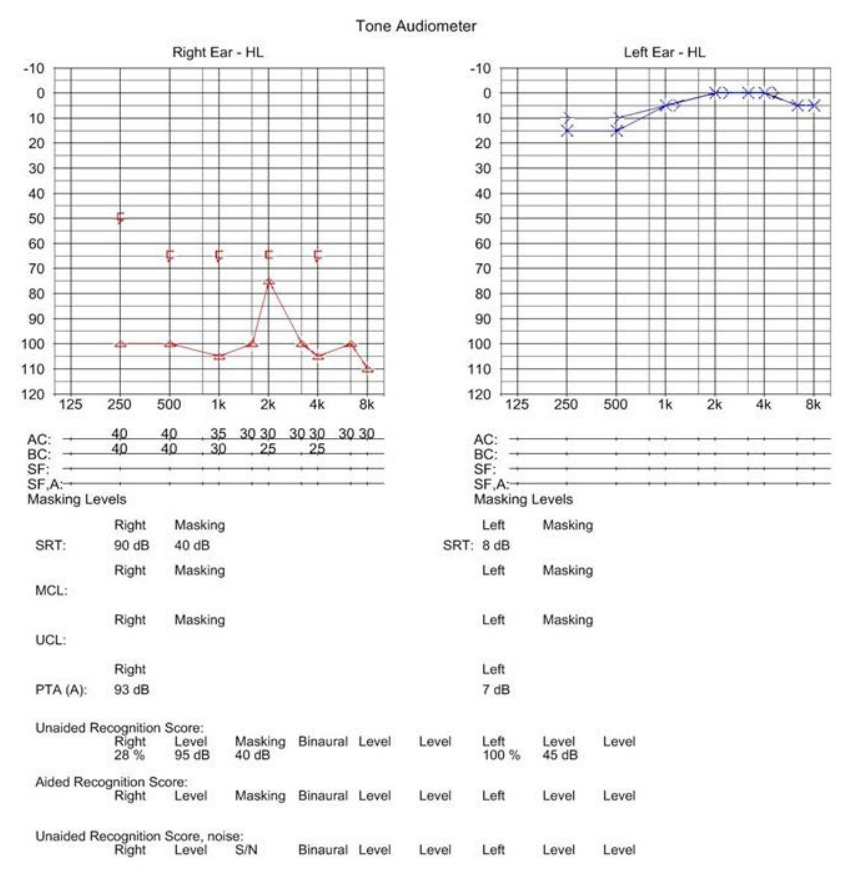

Figure 2. Pure tone audiometry of the patient: normal ranges of speech recognition threshold and pure tone average are noted in the left ear, but severe sensory neuronal hearing impairment is noted in the right ear, with a speech recognition threshold of 90 $\mathrm{dB}$ and a pure tone average of $93 \mathrm{~dB}$. 
This malformation is thought to be caused by the arrest of cochlear development during the fourth to seventh weeks of gestation and Mondini-like dysplasia was reported to be associated with mutation of the SLC26A4 gene, which codes for the membrane transport protein pendrin. In most cases, Mondini-like dysplasia occurs alone, but some cases occur in association with congenital cytomegalovirus infection, Klippel-Feil syndrome, Pendred syndrome, or DiGeorge Syndrome..$^{2,3,5,6}$ In this case, there was no specific finding suspicious of other comorbidities.

Clinical features of Mondini-like dysplasia include varying degrees of SNHL, CSF otorrhea or rhinorrhea, and recurrent meningitis, and are sometimes accompanied by vestibular vertigo. However, most patients may not have noticeable symptoms and signs. SNHL by Mondini-like dysplasia can be uni- or bilateral, but hearing is preserved to some extent in some cases..$^{2,5,6,8}$ In children with unknown SNHL, imaging tests such as HRCT or magnetic resonance imaging can provide important diagnostic clues to identify the cause of hearing impairment. HRCT of the temporal bones is the preferred modality for delineating anatomical pathology such as inner ear osseous dysplasia, including erosive or destructive lesions. ${ }^{10}$ Therefore, if IEMs are suspected, HRCT of temporal bones is the method of choice for evaluation., ${ }^{2,5-7}$

Mondini-like dysplasia is the most common congenital anomaly associated with CSF leakage with a temporal bone origin in children. ${ }^{5}$ Abnormal connections between the endolymphatic and perilymphatic space of the inner ear and subarachnoid space are observed in cases of Mondini-like dysplasia. This anatomical abnormality become CSF fistula. This condition is a risk factor for CSF contamination and can cause retrograde bacterial meningitis in patients with Mondini-like dysplasia. ${ }^{6}$ The most common infection-causing organism is $S$. pneumoniae, which colonizes the nasopharynx, and invades and settles into the upper and lower respiratory tract. ${ }^{5}$ In the present case, bacterial meningitis was caused by the middle ear infection that had spread to the CSF directly because $S$. pneumoniae was only cultured in CSF but not in blood.

Most cases of meningitis associated with Mondini-like dyspla-
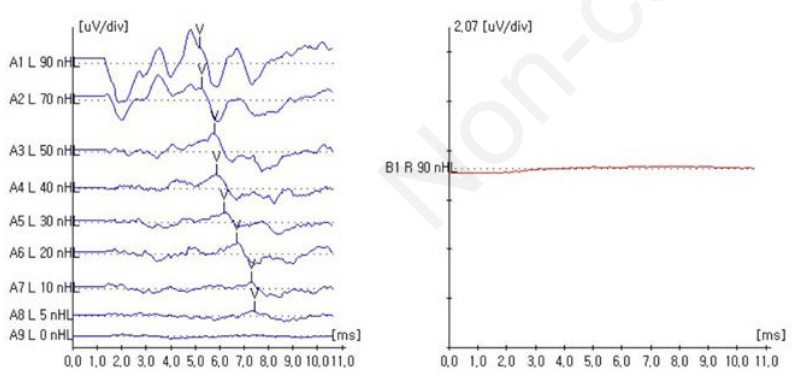

\begin{tabular}{|c|c|c|c|c|c|}
\hline \multicolumn{6}{|c|}{ Collection Parameters } \\
\hline $\begin{array}{l}\text { Wave } \\
\text { AI }\end{array}$ & $\begin{array}{l}\text { Transducer } \\
\text { Headphones }\end{array}$ & $\begin{array}{l}\text { Ear } \\
\text { Left }\end{array}$ & $\begin{array}{l}\text { Intensity } \\
99 \mathrm{~dB} \mathbf{~ n H L}\end{array}$ & $\begin{array}{l}\begin{array}{l}\text { Type } \\
\text { Click }\end{array} \\
\end{array}$ & $\begin{array}{l}\text { Frequency } \\
\text { N/A }\end{array}$ \\
\hline $\mathrm{A}_{2}$ & Headphones & Left & $70 \mathrm{~dB} \mathrm{nHL}$ & Click & $N / A$ \\
\hline${ }^{3}$ & Headphones & Left & $50 \mathrm{~dB}$ nHL & Click & $\mathrm{N} / \mathrm{A}$ \\
\hline A4 & Headphones & Left & $40 \mathrm{~dB}$ nHL & Click & $\mathrm{N} / \mathrm{A}$ \\
\hline A5 & Headphones & Left & 30dB nHL. & Click & $\mathrm{N} / \mathrm{A}$ \\
\hline A6 & Headphones & Left & $20 \mathrm{~dB} \mathrm{nHL}$ & Click & $\mathrm{N} / \mathrm{A}$ \\
\hline A7 & Headphones & Left & IOdB nHL & Click & $\mathrm{N} / \mathrm{A}$ \\
\hline A8 & Headphones & Left & $5 \mathrm{~dB} \mathrm{nHL}$ & Click & $\mathrm{N} / \mathrm{A}$ \\
\hline A9 & Headphones & Left & OdB nHL & Click & $\mathrm{N} / \mathrm{A}$ \\
\hline B1 & Headphones & Right & 90dB nHL. & Click & N/A \\
\hline
\end{tabular}

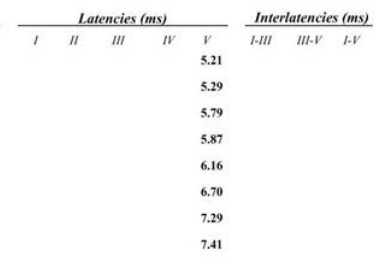

Figure 3. Auditory brainstem response analysis of the patient: normal responses of $\mathrm{V}$ wave latency and amplitude are noted in the left ear with an increase in the click stimulus rate, but no response is noted in the right ear despite 90 normal hearing level of click stimuli. sia are reported to occur in children less than 10 years old. However, Mondini-like dysplasia is not easily diagnosed before recurrent meningitis. ${ }^{2,5}$ In a previous case review, of 39 cases of Mondini-like dysplasia associated with meningitis, 36 patients were diagnosed after 2 to 20 recurrent meningitis episodes. ${ }^{6}$ Therefore, in patients with Mondini-like dysplasia, early diagnosis and successful surgical repair of the CSF leakage is very important to prevent the sequelae or recurrent episodes of meningitis. ${ }^{6,7}$ However, many pitfalls inhibit early diagnosis of Mondini dysplasia. In young children with unilateral malformation alone, it is difficult to recognize hearing problems early. Besides, the hearing impairment might be misinterpreted as resulting from preceding episodes of meningitis. There have been several reports where CSF leaks into the middle ear, producing a fluid level behind the tympanic membrane or bulging, were initially misinterpreted as being benign serous otitis media. ${ }^{2}$ Emergency physicians should suspect inner ear pathologies after confirmation of the patient's past history of meningitis or hearing impairment. We strongly suspected inner ear pathologies based on the fact that the patient had a history of ipsilateral hearing impairment. We took an HRCT of the temporal bones of the patient and were finally able to diagnose the patient's Mondini-like dysplasia at the first meningitis episode.

In the treatment of meningitis associated with congenital anomalies, such as Mondini-like dysplasia, the most critical points are early diagnosis and proper surgical repair of CSF fistula. ${ }^{7}$ Exploratory tympanotomy with obliteration of the defect and middle ear cavity is recommended as an initial treatment choice of CSF fistula associated with the congenital anomaly. In cases where the extracranial procedure had failed, it has been reported that intracranial procedures for repairing the defect using temporal craniotomy with an exploration of the middle cranial fossa are effective. In addition, lumboperitoneal shunting procedures have been reported as effective adjunctive therapy options to control CSF leakage associated with impaired CSF absorption in congenital anomalies of the ear. ${ }^{11,12}$ In our case, the patient underwent external auditory canal obliteration via subtotal petrosectomy three months after meningitis was cured.

\section{Conclusions}

Our case provides valuable insights toward an early diagnosis of Mondini-like dysplasia, at a point in the care continuum that can include the time period of provision of care in the ED. The case gives rise to two suggestions for emergency physicians. First, assessment of hearing function upon recovery by children who have had an episode of meningitis is crucial. Although hearing impairment is the most common sequela of meningitis, abnormal hearing test results not only show us the post-meningitis functional status of patients but also provide insights into whether inner ear malformations exist or not. Second, a history of persistent otorrhea and/or rhinorrhea should be evaluated to confirm whether the fluids are of CSF origin or not. If CSF otorrhea or rhinorrhea with unilateral hearing impairment is present, an imaging study should be performed with suspicion of inner ear malformation. HRCT of the temporal bones is the test of choice for inner ear malformations. Prophylactic antibiotic treatment has not been proven to be useful toward prevention of recurrent meningitis, but surgical intervention has value toward preventing such recurrences. 


\section{References}

1. Durand ML, Calderwood SB, Weber DJ, et al. Acute bacterial meningitis in adults - A review of 493 episodes. N Engl J Med 1993;328:21-8.

2. Tebruegge M, Curtis N. Epidemiology, etiology, pathogenesis, and diagnosis of recurrent bacterial meningitis. Clin Microbiol Rev 2008;21:519-37.

3. Schuknecht HF. Mondini dysplasia: a clinical and pathological study. Ann Otol Rhinol Laryngol 1980;89:3-23.

4. Anandi S, Tullu MS, Bhatia S, Agrawal M. Mondini dysplasia as a cause for recurrent bacterial meningitis: an early diagnosis. J Child Neurol 2012;27:1052-5.

5. Çiftdoğan DY, Bayram N, Özdemir Y, et al. A case of Mondini dysplasia with recurrent Streptococcus pneumoniae meningitis. Eur J Pediatr 2009;168:1533.

6. Ohlms LA, Edwards MS, Mason EO, et al. Recurrent meningitis and Mondini dysplasia. JAMA Otolaryngol 1990;116:608-12.

7. Stevenson D, Proops D, Phelps P. Severe cochlear dysplasia causing recurrent meningitis: a surgical lesson. J Laryngol Otol 1993;107:726-9.

8. Zheng Y, Schachern PA, Cureoglu S, et al. The shortened cochlea: its classification and histopathologic features. Int $\mathrm{J}$ Pediatr Otorhinolaryngol 2002;63:29-39.

9. Sennaroğlu L, Bajin MD. Classification and current management of inner ear malformations. Balkan Med J 2017;34:397411.

10. Mafong DD, Shin EJ, Lalwani AK. Use of laboratory evaluation and radiologic imaging in the diagnostic evaluation of children with sensorineural hearing loss. Laryngoscope 2002;112:1-7.

11. Herther C, Schindler RA. Mondini's dysplasia with recurrent meningitis. Laryngoscope 1985;95:655-8.

12. Park TS, Hoffman HJ, Humphreys RP, Chuang SH. Spontaneous cerebrospinal fluid otorrhea in association with a congenital defect of the cochlear aqueduct and Mondini dysplasia. Neurosurgery 1982;11:356-62. 